\title{
Soil porosity distribution representative elementary area analyzed through gamma-ray computed tomography**
}

\author{
Talita Rosas Ferreira ${ }^{1 *}$ and Luiz Fernando Pires ${ }^{2}$ \\ ${ }^{1}$ Physics Graduate Program, ${ }^{2}$ Laboratory of Soil Physics and Environmental Sciences, \\ Department of Physics, State University of Ponta Grossa (UEPG), Av. Carlos Cavalcanti, 4748, CEP 84.030-900, \\ Ponta Grossa, PR, Brazil
}

Received May 3, 2016; accepted September 29, 2016

A b s t r a c t. This study aims to use $\gamma$-ray computed tomography images to define the representative elementary area of the soil porosity distribution. Different textured soils from Southeast Brazil and different schemes of areas were analyzed. The image acquisition was performed in 2006 using a first generation $\gamma$-ray computed tomography system equipped with ${ }^{241} \mathrm{Am}$ radioactive source. Consecutive increasing areas located at the center (scheme 1), the inferior (scheme 2) and superior (scheme 3 ) borders of the sample computed tomography image, with size areas ranging from 1.2 to $678.8 \mathrm{~mm}^{2}$, were selected. The full width at a half maximum parameter was used to describe the samples soil porosity distribution and mathematical analysis concepts were adopted to define the representative elementary area. The representative elementary areas found for the sandy soil (Geric Ferralsol) and clayey soils (Rhodic Ferralsol and Eutric Nitosol) were respectively: $514.3,514.3$ and $555.4 \mathrm{~mm}^{2}$ (scheme 1); 279.5, 393.3 and $457.4 \mathrm{~mm}^{2}$ (scheme 2); and 457.4, 457.4 and $457.4 \mathrm{~mm}^{2}$ (scheme 3 ). The results confirmed that the representative elementary area were influenced by the soil texture and management. Different schemes were noticed to provide different representative elementary areas for the same soil, which suggests that this procedure was efficient to detect the heterogeneity inside the soil samples.

K e y w o r d s: image analysis, soil structure, soil porous system, representative measurements

\section{INTRODUCTION}

Total porosity $(\varphi)$ is a measure of the soil porous space and refers to an index of the relative pore space in a soil. This soil physical property is defined by the ratio of the pore volume to the total volume of a representative sam-

*Corresponding author e-mail: tali.rf@gmail.com

**The authors acknowledge CNPq (National Council for Scientific and Technological Development) for the provision of the productivity grant in research (304310/2011-5) and CAPES (Coordination for the Improvement of Higher Education Personnel ) for the M.S. scholarship. ple (Jury and Horton, 2004). According to Hillel (1998), $\varphi$ should be equal to the areal porosity, related to the fraction of pores in a representative 2D cross-section area.

However, any $\varphi$ measurement should be representative of the porous medium. The soil represents a heterogeneous porous system with $\varphi$ varying in the space, but also as a function of the measure scale (Borges et al., 2012; VandenBygaart and Protz, 1999). Small samples cannot present representative values of $\varphi$ (domain of microscope effects). However, the increase of the sample size makes the fluctuations in the $\varphi$ determination small (domain of porous medium), and a consistent measure of this physical parameter is obtained (Bear and Cheng, 2010). Therefore, from a particular minimum size the microscopic effects become insignificant and the relevant variable takes a value which fluctuates slightly around an average value (Baveye and Boast, 1999; Bear, 1972).

For 2D images, the representative minimum sample size is known as the representative elementary area $(R E A)$. The $R E A$ can initially be determined by considering the porous medium areas, gradually larger, around a certain point, and then calculating the relevant variable in these areas. $R E A$ analyses are specially interesting for measurements of soil physical properties employing micromorphological or tomography evaluations (Borges and Pires, 2012; VandenBygaart and Protz, 1999).

Computed tomography (CT) represents a technique based on the principle of radiation attenuation. Gamma-ray CT ( $\gamma$-ray CT) provides 2D images of transversal sections of objects with millimetric and micrometric spatial resolutions. When compared to conventional methods, the greatest advantage of $\gamma$-ray CT lies in the fact that, depending

(C) 2016 Institute of Agrophysics, Polish Academy of Sciences 
T a b l e 1. Properties of the investigated soils collected from different sites

\begin{tabular}{|c|c|c|c|c|c|c|}
\hline \multirow{2}{*}{ Soil } & \multirow{2}{*}{ Collect place } & Sand & Silt & Clay & \multirow{2}{*}{ Texture } & \multirow{2}{*}{$\begin{array}{c}\rho_{p} \\
\left(\mathrm{~g} \mathrm{~cm}^{-3}\right)\end{array}$} \\
\hline & & & $(\%)$ & & & \\
\hline GF & Sertãozinho $\left(22^{\circ} 43^{\prime} 12^{\prime \prime} \mathrm{S} / 47^{\circ} 36^{\prime} 32^{\prime \prime} \mathrm{W}\right)$ & 66 & 6 & 28 & $\begin{array}{c}\text { Sandy clay } \\
\text { loam }\end{array}$ & 2.55 \\
\hline $\mathrm{RF}$ & $\operatorname{IAC}\left(22^{\circ} 41^{\prime} 22^{\prime \prime} \mathrm{S} / 47^{\circ} 38^{\prime} 40^{\prime \prime} \mathrm{W}\right)$ & 26 & 26 & 48 & Clayey & 2.54 \\
\hline EN & Areão $\left(22^{\circ} 51^{\prime} 52^{\prime} " \mathrm{~S} / 47^{\circ} 38^{\prime} 40^{\prime \prime} \mathrm{W}\right)$ & 24 & 33 & 43 & Clayey & 2.68 \\
\hline
\end{tabular}

GF - Geric Ferralsol, RF - Rhodic Ferralsol, EN - Eutric Nitosol, $\rho_{p}-$ soil particle density; size fractions: sand (2.00-0.02 mm), silt $(0.02-0.002 \mathrm{~mm})$ and clay $(<0.002 \mathrm{~mm})$ according to ISSS system.

on the spatial resolution attained, over 4000-5000 density/ porosity values can be obtained from the tomography unit matrix (Crestana et al., 1992). This large amount of data is commonly used to reconstruct the 2D image in first generation $\gamma$-ray CT scanners (Ferreira et al., 2015; Pedrotti et al., 2005; Timm et al., 2005; Vaz et al., 1989).

Conventional methods for density evaluation such as the paraffin sealed clod or volumetric ring utilize bulk samples and only one measurement of this property is obtained for each sample (Jury and Horton, 2004). Through the knowledge of the particle density it is possible to derive the soil porosity (Hillel, 1998).

Since the $\gamma$-ray CT allows to acquire punctual information over a cross section of a sample, it is sensitive enough to evaluate variations in the soil structure due to natural and artificial processes. Besides that, $\gamma$-ray CT consists of an ideal technique to study $R E A$, considering that this technique makes it possible to analyze particular sets of data, which can represent for instance transects or sub-areas, inside the transversal sections of the sample. In comparison with the micromorphologic technique, which could also be used for REA determination by image analyses, the $\gamma$-ray CT presents advantages due to its non-destructive analysis nature. Another advantage is the no need of any sample pre-treatment, which allows the reutilization of the studied material (Pires et al., 2010).

The main objective of this study was to use the $\gamma$-ray $\mathrm{CT}$ technique to define $R E A$ from the soil sample porosity distribution analysis. It proposes to analyze whether different soils can present different $R E A$ values and whether different construction schemes of neighboring areas in the CT image present different REA values. The last result is interesting because it allows the evaluation of the soil spatial variability effect when defining REA. The use of CT is also interesting because a large amount of data to evaluate the soil porosity distribution can be obtained through it.

\section{MATERIAL AND METHODS}

The eighteen soil samples analyzed in this study were collected from three different sampling sites located in Piracicaba (22 $40^{\prime} 22^{\prime \prime} \mathrm{S} ; 47^{\circ} 38^{\prime} 40^{\prime}$ "W; $580 \mathrm{~m}$ a.s.l) in the
Southeast region of Brazil. Six undisturbed samples classified as Rhodic Ferralsol (RF) were collected from the IAC experimental farm; six classified as Geric Ferralsol (GF) from the Sertãozinho experimental farm; and another six classified as Eutric Nitosol (EN) (FAO/ISRIC/ISSS, 1998) from the Areão experimental farm in 2006 (Table 1). The last two experimental units belong to the "Luiz de Queiroz" College of Agriculture (ESALQ).

The three studied soils represent important groups employed in the Brazilian agricultural production. GF samples were collected in a non-cultivated area covered by grass; samples of RF in a native mixed forest; and EN from a coffee field established in 2001. Table 1 presents the percentage amounts of sand, silt and clay fractions of the three mentioned soils, as well as their textural classification. The soils were classified as clayey (EN and RF) and sandy clay loam (GF) (Lal and Shukla, 2004). However, throughout the text the sandy clay loam soil will be referred to as sandy.

The undisturbed samples $(3.0 \mathrm{~cm}$ high and $4.8 \mathrm{~cm}$ inner diameter) were collected at a depth of $0-10 \mathrm{~cm}$ using volumetric rings. The sampler was inserted into the soil through impact, with a rubber hammer falling from a fixed height, as traditionally done. After complete insertion of the volumetric rings into the soil, the surrounding soil was carefully removed to minimize further soil disturbance due to vibration, shear stress, and compaction. The excess soil outside the cylinder was carefully trimmed off and top and bottom surfaces of the samples were made flat to be sure that the soil volume was equal to the internal volume of the soil.

Since the soil water content is very important at the sampling time to minimize impact effects, samples were collected near their plastic limit, about three days after a high intensity rainfall event. In order to lessen the effects of spatial variability, the soil samples were collected $10 \mathrm{~cm}$ far one from another.

The $\gamma$-ray CT used in this study is a first generation scanning type, developed at the EMBRAPA/CNPDIA, which uses a fixed arrangement of source and detector, with movements of rotation and translation of the sample, and a parallel beam (Fig. 1). The radioactive source is ${ }^{241} \mathrm{Am}$ 

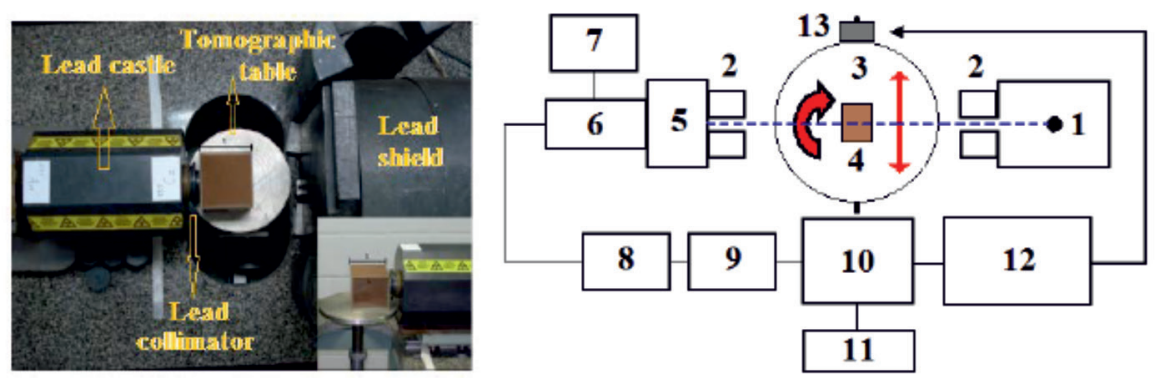

Fig. 1. Picture of the $\gamma$-ray CT scanner and its schematic diagram: 1 - radioactive source, 2 - lead collimator, 3 - tomographic table, 4 - soil sample, 5 - NaI(Tl) scintillation detector, 6 - photomultiplier tube, 7 - high voltage unit, 8 - amplifier, 9 - single channel analyzer, 10 - counter, 11 - timer, 12 - PC, 13 - stepper motor controls. Adapted from Pires et al. (2002, 2011a).

(3.7 GBq/59.54 keV). Lead collimators with $1.0-4.5 \mathrm{~mm}$ diameter openings were fixed at the source output and the detector input, respectively, aiming to prevent the detection of secondary photons.

In the CT scanner, the rotation-translation system with step motors permits a maximum translation of $20 \mathrm{~cm}$. The gamma detector used is an encapsulated, thallium doped, sodium iodide crystal $\mathrm{NaI}(\mathrm{Tl})$ of a plain type with $7.62 \times 7.62 \mathrm{~cm}$ dimensions. The detector is suitably shielded from external radiation with lead (Fig. 1), optically coupled to a conventional system of photomultiplier tube (Ortec-276), high-voltage unit (Ortec-556), amplifier (Ortec-572), single-channel analyzer (Ortec-550A), counter and timer (Ortec-994), modular bin (Ortec-4001A) and microcomputer (Intel Pentium-100). The description of a similar scanner can be found in Cruvinel et al. (1990).

The evaluation of soil physical properties using CT depends on the calibration of the system, which basically consists in finding a relation between the tomography unit $(T U)$ and the linear attenuation coefficient $(\kappa)$ for some homogeneous materials. The $\kappa$ of each material can be experimentally determined using the Beer-Lambert law (Eq. (1)), which provides the relation between the transmitted photons $(I)$ by the absorber, of thickness $x$, and the transmitted photons without its presence $\left(I_{0}\right)$. In general, the $\kappa$ value obtained represents an average of several measurements of the material taken in different positions, while the values of $T U$ are extracted from the $\mathrm{CT}$ images (Pires et al., 2011a). A detailed description of first generation $\gamma$-ray CT system calibration process can be found in the literature (Crestana et al., 1992):

$$
I=I_{0} e^{(-\kappa x)} \therefore \kappa=\frac{1}{x} \ln \left(\frac{I_{0}}{I}\right)
$$

Thus, the following material samples were used to calibrate the $\gamma$-ray CT system: acrylic, ethanol, water, nylon, glycerin and soils (GF, RF and EN) (Fig. 2). It is important to mention that, in order to ensure the homogeneity of the disturbed soil samples, they were slightly ground

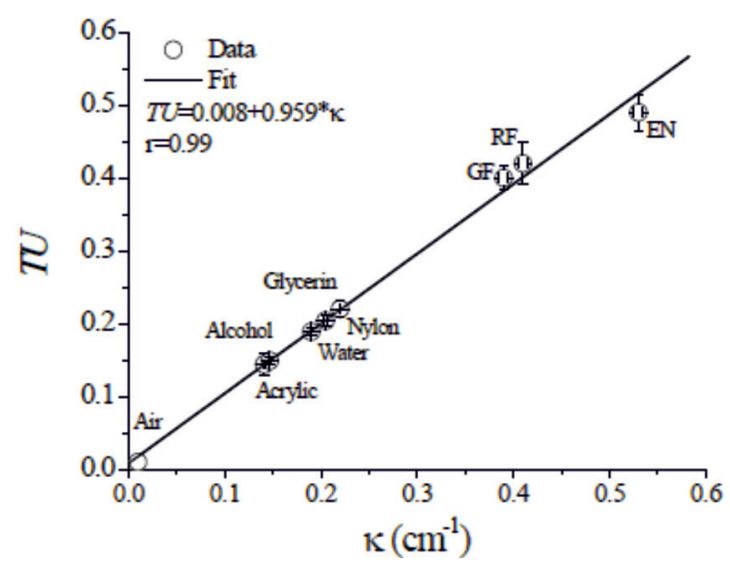

Fig. 2. CT calibration curve for ${ }^{241} \mathrm{Am}$ gamma-ray radioactive source. Error bars represent the measurement standard deviation. The value 0.959 is the parameter $\alpha$ (inclination of straight line) of Eq. (2), which relates the tomography unit (TU) and the soil physical properties.

and reduced to diameters lower than $1 \mathrm{~mm}$ (Pires et al., $2011 b)$. The mass attenuation coefficient $(\mu)$ values, which are defined by the ratio between the linear attenuation coefficient and the material density, obtained for each soil and water, were 0.2492 (GF), 0.3064 (RF), 0.3280 (EN) and $0.1989 \mathrm{~cm}^{2} \mathrm{~g}^{-1}$ (water). These values are in accordance with those found in the literature (Ferraz and Mansell, 1979).

The total measuring time for each tomographic scan was around $47 \mathrm{~h}$. The total counts were taken for sufficient time periods ( $26 \mathrm{~s}$ ) to obtain statistical accuracy better than $1 \%$ without any absorber and around 3\% with absorber (Knoll, 2010; Turner et al., 2012). In order to reduce the effect of background radiation, the detector was surrounded by lead (10 cm thick) (Fig. 1). Only a small aperture was maintained in front of the radioactive source to detect $I$ and $I_{0}$ through the soil. Due to the period of time (26 s) selected for each measurement and the detector lead shield, the background radiation correction was unnecessary.

Samples were fixed on the measurement table with the help of adhesive tape in order to prevent any movements during scanning. The samples (Fig. 3) scanning were 

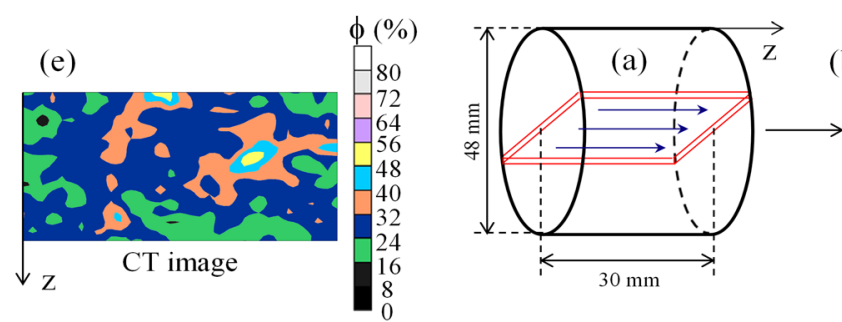

(d) \begin{tabular}{l|l|l|l|l|l|l|l|l|l|l|l|l|}
39 & 37 & 39 & 42 & 48 & 51 & 52 & 46 & 43 & 38 & 42 & 50 & 57 \\
\hline
\end{tabular} \begin{tabular}{lllllll|l|l|l|l|l|l|l}
45 & 44 & 37 & 41 & 43 & 53 & 54 & 46 & 38 & 38 & 37 & 46 & 47
\end{tabular} \begin{tabular}{ll|l|l|l|l|l|l|l|l|l|l|l}
47 & 45 & 42 & 45 & 47 & 45 & 52 & 47 & 39 & 40 & 46 & 47 & 46
\end{tabular} \begin{tabular}{|l|l|l|l|l|l|l|l|l|l|l|l|l|l|}
41 & 42 & 44 & 50 & 46 & 42 & 44 & 47 & 46 & 48 & 46 & 50 & 48 \\
\hline
\end{tabular} \begin{tabular}{llllllllll|l|l|l|l}
40 & 43 & 47 & 49 & 43 & 44 & 45 & 51 & 54 & 48 & 42 & 41 & 43
\end{tabular} \begin{tabular}{|l|l|l|l|l|l|l|l|l|l|l|l|l|}
39 & 39 & 45 & 42 & 35 & 44 & 46 & 51 & 49 & 48 & 46 & 41 & 46
\end{tabular} \begin{tabular}{ll|l|l|l|l|l|l|l|l|l|l|l|}
34 & 34 & 42 & 47 & 40 & 44 & 41 & 39 & 40 & 46 & 50 & 44 & 47
\end{tabular} $\phi$ matrix $(17 \times 33)$, Eq. 4 \begin{tabular}{l|l|l|l|l|l|l|l|l|l|l|l|l|l|l|l|l|}
0 & 0 & 0 & 0 & 0 & 0 & 0 & 0 & 0 & 0 & 0 & 0 & 0 & 0 & 0 & 0 & 0
\end{tabular}

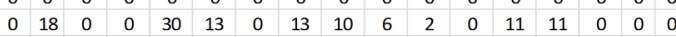
$\begin{array}{llllllllllllllllllll}\text { (b) } & 0 & 0 & 455 & 467 & 454 & 428 & 384 & 367 & 359 & 398 & 421 & 458 & 435 & 375 & 323 & 31 & 0\end{array}$

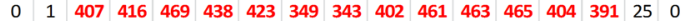

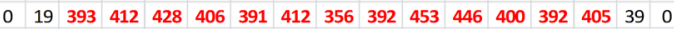
\begin{tabular}{l|l|l|l|l|l|l|l|l|l|l|l|l|l|l|l|l}
0 & 25 & 441 & 428 & 414 & 374 & 404 & 430 & 419 & 393 & 404 & 387 & 398 & 374 & 385 & 22 & 0
\end{tabular}

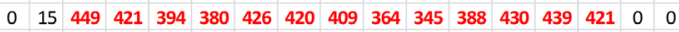
$\begin{array}{llllllllllllllllllll}0 & 22 & 450 & 454 & 409 & 432 & 485 & 417 & 403 & 362 & 381 & 384 & 404 & 438 & 399 & 36 & 0\end{array}$ $\begin{array}{lllllllllllllllllll}0 & 13 & 490 & 489 & 431 & 396 & 445 & 413 & 440 & 454 & 444 & 399 & 375 & 413 & 395 & 7 & 0\end{array}$ \begin{tabular}{l|l|l|l|l|l|l|l|l|l|l|l|l|l|l|l|l}
0 & 0 & 0 & 0 & 18 & 0 & 25 & 5 & 22 & 6 & 41 & 10 & 19 & 19 & 9 & 1 & 0 \\
\hline
\end{tabular}

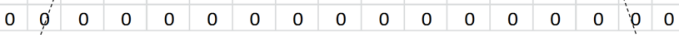
$T U$ matrix $(80 \times 80)$


\begin{tabular}{|l|l|l|l|l|l|l|l|l|l|l|l|l|}
1.39 & 1.42 & 1.60 & 1.50 & 1.44 & 1.19 & 1.17 & 1.37 & 1.57 & 1.58 & 1.59 & 1.38 & 1.34
\end{tabular} \begin{tabular}{|l|l|l|l|l|l|l|l|l|l|l|l|l|}
\hline 1.34 & 1.41 & 1.46 & 1.39 & 1.34 & 1.41 & 1.22 & 1.34 & 1.55 & 1.52 & 1.37 & 1.34 & 1.38 \\
\hline
\end{tabular} \begin{tabular}{|l|l|l|l|l|l|l|l|l|l|l|l|l|}
1.51 & 1.46 & 1.41 & 1.28 & 1.38 & 1.47 & 1.43 & 1.34 & 1.38 & 1.32 & 1.36 & 1.28 & 1.32
\end{tabular} \begin{tabular}{|l|l|l|l|l|l|l|l|l|l|l|l|l|}
1.53 & 1.44 & 1.35 & 1.30 & 1.46 & 1.43 & 1.40 & 1.24 & 1.18 & 1.33 & 1.47 & 1.50 & 1.44
\end{tabular} \begin{tabular}{|l|l|l|l|l|l|l|l|l|l|l|l|l|l|}
\hline 1.54 & 1.55 & 1.40 & 1.48 & 1.66 & 1.42 & 1.38 & 1.24 & 1.30 & 1.31 & 1.38 & 1.50 & 1.36
\end{tabular}


$\rho_{\mathrm{s}}$ matrix $(17 \times 33)$, Eq. 3


Fig. 3. (a) Schematic representation of the CT scanning along the central region of the soil sample; (b) tomography unit (TU) matrix obtained after the reconstruction procedure. The highlighted numbers in red represent the sub-matrix selected for $R E A$ analysis; the number 0 represents the air attenuation. (c) $T U$ sub-matrix converted to a soil bulk density $\left(\rho_{s}\right)$ matrix by Eq. (3). (d) $\rho_{s}$ matrix converted to a soil total porosity $(\varphi)$ matrix by Eq. (4); (e) CT image in $\varphi$ color scale; (f) schemes 1,2 and 3 of consecutive area selection; (g) curve adjustment equation and schematic representation of the parameters utilized to calculate the full width at half maximum $(F W H M)$ (Downing and Clark, 2000); (h) graphs of frequency distribution for small (06), intermediate (12) and large (17) area sizes, for one of the studied samples and schemes; (i) FWHM graphs as a function of the different areas selected, according to schemes 1, 2 and 3 , for one of the studied samples. The dotted line represents the FWHM of the last area (LA), which is the same for all schemes; red filled icon represents the $R E A$ related to the $10 \%$ relative deviation. 
performed up to a total rotation of $180^{\circ}$, with $2.25^{\circ}$ angular steps and $1.1 \mathrm{~mm}$ linear steps, resulting in 80 projections. The image acquisition planes were located in the central part of the samples, in the vertical direction, along their depth (Fig. 3a).

Tomography unit $80 \times 80$ data matrices, equivalent to $6400 T U$ values, representing one full cross-section of each sample $(30 \times 48 \mathrm{~mm})$ under study, were obtained after the reconstruction procedure (Fig. 3b). The filtered back projection method through the program Microvis (2000) was employed for image reconstruction. The size of the pixel obtained was $1.1 \times 1.1 \mathrm{~mm}$, which was calculated taking the ratio between the soil sample diameter and the number of pixels in the reconstruction matrix.

The largest common area to all samples, equivalent to a $17 \times 33$ sub-matrix $(18.7 \times 36.3 \mathrm{~mm})$, was identified and marked in the full $T U$ matrices $(80 \times 80)$ in order to avoid the regions close to the ring border (Fig. 3b). The area size was obtained by multiplying the sub-matrix size by the linear step value. The borders were avoided due to the possible presence of artifacts in the interface sampleair/sample-ring, which might affect the material physical property analysis via CT (Cruvinel et al., 1990; Kak and Slaney, 1999). In addition, this procedure ensured that the regions of possible sampling disturbance were properly disregarded.

Different $\mu$ values are obtained for each path crossed by the radiation beam interacting with the soil, because $\mu$ is influenced by the mineral particle, organic matter, water and the air contribution (Pires et al., 2005). TU is directly related to $\mu$. For this reason, when the soil is considered as an absorber medium, the relation between the $T U$ and the soil physical properties is given by:

$$
T U=\alpha \kappa \therefore T U=\alpha\left(\mu_{s} \rho_{s}+\mu_{w} \rho_{w} \theta\right),
$$

where: $\alpha$ is a parameter obtained from the correlation between $T U$ and $\kappa$ of the materials used in the CT calibration (Fig. 2), $\mu_{s}$ and $\mu_{w}\left(\mathrm{~cm}^{2} \mathrm{~g}^{-1}\right)$ are the soil and water mass attenuation coefficients, respectively, $\rho_{s}$ and $\rho_{w}\left(\mathrm{~g} \mathrm{~cm}^{-3}\right)$ are the soil bulk and water densities and $\theta$ is the volumetric water content.

It is important to say that $T U$ takes the air as the medium with the minimum possible $\kappa$ value. It is related to the hounsfield unit (HU) that takes water as a reference media for which $\mathrm{HU}=0$. Due to this fact, the attenuation of the beam by the air is neglected in Eq. (2). When the $\gamma$-ray beam interacts with the air during CT scanning, the $T U$ values are equal to zero, since the air is considered the reference medium.

So, dry soil bulk density can then be calculated for each pixel of the CT data matrix through:

$$
\rho_{s}=\frac{1}{\mu_{s}}\left(\frac{T U}{\alpha}\right) .
$$

Therefore, the $T U$ sub-matrices are initially converted into $\rho_{s}$ matrices (Fig. 3c) and, later on, knowing the particle density $\left(\rho_{p}\right)$ of each studied soil (Table 1), into $\varphi$ matrices (Fig. 3d, e) according to:

$$
\phi=\left(1-\frac{\rho_{s}}{\rho_{p}}\right) .
$$

In order to determine $R E A$ as a function of the $\varphi$ distribution, areas were selected from the center of $\varphi$ matrices (scheme 1), without extrapolating the maximum area previously selected. In the same way, consecutive areas from the matrix lower border (scheme 2) and from the upper border (scheme 3) were also selected, respectively (Fig. 3f). The initial area obtained, in all cases, corresponded to a $1 \times 1$ $(1.1 \times 1.1 \mathrm{~mm})$ square matrix. Table 2 presents the selected area sizes for the schemes employed in this study. The relevance of studying the $R E A$ behaviour, according to the different area selection schemes described, lies in the fact that this allows to evaluate whether the $\varphi$ sample heterogeneity can affect the $R E A$ definition.

The $R E A$ estimate was established based on Vanden Bygaart and Protz (1999) and Borges et al. (2012) studies. Porosity distributions within each area followed by the construction of $\varphi$ frequency graphs through the CT image (\%) were obtained. This procedure was carried out for each of the areas and soil samples $(\mathrm{n}=18)$. It was observed that from the 5th selected area, for the three selection schemes (Fig. 3f), the $\varphi$ frequency distribution contained in each area was well represented by a Gaussian distribution (see curve adjustment equation in Fig. $3 \mathrm{~g}$ ), since the coefficients of determination $\left(\mathrm{R}^{2}\right)$ obtained indicated good agreement of data. Therefore, Gaussian adjustments were performed in these graphs, starting at each sample 5th area, which provided the full width at half maximum (FWHM) parameter for the distributions obtained (Fig. 3h).

The $R E A$ was defined when the relative deviation $(R D)$ between the $F W H M$ value corresponding to the $\varphi$ frequency distribution of the last area $\left(F W H M_{L A}\right)$ and the $F W H M$ value obtained for each of the selected areas in the $\varphi$ matrices was not over $10 \%$ (Eq. (5)):

$$
R D=\frac{F W H M_{L A}-F W H M}{F W H M_{L A}} 100 .
$$

when at least three consecutive areas, excluding the last area, did not differ between themselves regarding the $10 \%$ $R D$, the $R E A$ was reached (Fig. 3i).

\section{RESULTS AND DISCUSSION}

Figure 4 present the results of the REA definition for one sample of each group of samples, used as an example of the studied soils. For the GF soil, the CT image in color scale (Fig. 4Aa) highlights, qualitatively, the $\varphi$ distribution 
T a b l e 2. Size of each area (height $\times$ width) selected inside the total soil porosity $(\varphi)$ matrix according to schemes 1,2 and 3

\begin{tabular}{|c|c|c|c|c|c|}
\hline Area & Size $\left(\mathrm{mm}^{2}\right)$ & Area & Size $\left(\mathrm{mm}^{2}\right)$ & Area & Size $\left(\mathrm{mm}^{2}\right)$ \\
\hline \multicolumn{6}{|c|}{ Scheme 1} \\
\hline 01 & $1.1 \times 1.1=1.2$ & 07 & $14.3 \times 14.3=204.5$ & 13 & $18.7 \times 27.5=514.3$ \\
\hline 02 & $3.3 \times 3.3=10.9$ & 08 & $16.5 \times 16.5=272.3$ & 14 & $18.7 \times 29.7=555.4$ \\
\hline 03 & $5.5 \times 5.5=30.3$ & 09 & $18.7 \times 18.7=349.7$ & 15 & $18.7 \times 31.9=596.5$ \\
\hline 04 & $7.7 \times 7.7=59.3$ & 10 & $18.7 \times 20.9=390.8$ & 16 & $18.7 \times 34.1=637.7$ \\
\hline 05 & $9.9 \times 9.9=98.0$ & 11 & $18.7 \times 23.1=432.0$ & 17 & $18.7 \times 36.3=678.8$ \\
\hline 06 & $12.1 \times 12.1=146.4$ & 12 & $18.7 \times 25.3=473.1$ & - & - \\
\hline \multicolumn{6}{|c|}{ Schemes 2 and 3} \\
\hline 01 & $1.1 \times 1.1=1.2$ & 07 & $7.7 \times 14.3=110.1$ & 13 & $14.3 \times 27.5=393.3$ \\
\hline 02 & $2.2 \times 3.3=7.3$ & 08 & $8.8 \times 16.5=145.2$ & 14 & $15.4 \times 29.7=457.4$ \\
\hline 03 & $3.3 \times 5.5=18.2$ & 09 & $9.9 \times 18.7=185.1$ & 15 & $16.5 \times 31.9=526.4$ \\
\hline 04 & $4.4 \times 7.7=33.9$ & 10 & $11.0 \times 20.9=229.9$ & 16 & $17.6 \times 34.1=600.2$ \\
\hline 05 & $5.5 \times 9.9=54.5$ & 11 & $12.1 \times 23.1=279.5$ & 17 & $18.7 \times 36.3=678.8$ \\
\hline 06 & $6.6 \times 12.1=79.9$ & 12 & $13.2 \times 25.3=334.0$ & - & - \\
\hline
\end{tabular}

inside the region corresponding to the largest area $(17 \times 33$ sub-matrix) selected (Table 2). The FWHM obtained for three areas of different sizes (scheme 1) was almost similar, which means that the $\varphi$ value interval comprised by these three areas was practically the same (Fig. 4Ab).

Therefore, the enlargement of areas from the center might influence the maximum frequency of $\varphi$ values shown to be in them, but not the $\varphi$ value interval, that is, the FWHM. Such stable behaviour was also observed in areas selected according to schemes 2 and 3 (Fig. 4Ac). However, the REA was not reached for scheme 3 , because the $10 \% R D$ was not satisfied in a specific area (14) and the minimum set of three consecutive areas was not fulfilled.

For the RF soil (Fig. 4B), it is possible to observe through the $\mathrm{CT}$ image (Fig. 4Ba) a higher heterogeneity than that presented for the GF. Figure $4 \mathrm{Bb}$ (scheme 1) shows that the larger the area selected is the larger the FWHM curve obtained is for this soil, that is, more heterogeneous are the $\varphi$ values comprised. In the meanwhile, the maximum value for the $\varphi$ appearance frequency shows small changes between an intermediary area and the last one. The CT image (Fig. 4Ba) shows indeed that, when the area increases from the center of the image, the distribution of $\varphi$ values becomes more heterogeneous, as different $\varphi$ values start to be accounted for, increasing the $F W H M$ value.

Regarding schemes 1 and 2 (Fig. 4Bc), the FWHM starts with low values and grows gradually without reaching a plateau, making it impossible to define the REA for the
$10 \% R D$ established. Considering scheme 3, the FWHM oscillates from the initial to the intermediary areas reaching a plateau, satisfying the $10 \% R D$. Thus, the area selection from the upper border reveals a more homogeneous $\varphi$ distribution than that found in areas selected from the lower border and center of the sample.

The behaviour previously identified in Fig. $4 \mathrm{Bb}$ is also observed between the initial and intermediate areas in Fig. $4 \mathrm{Cb}$ (EN, scheme 1). The FWHM of the curve obtained for the last area, in turn, is lower than that obtained for the intermediary area, while the $\varphi$ appearance frequency maximum value is higher. These observations are in accordance with the graph in Fig. 4Cc, for scheme 1, in which the $F W H M$ shows an increase in the intermediary areas and later on decreases. This means that the distribution of $\varphi$ values is more heterogeneous in a central area of intermediary size than that found in the largest area selected.

According to scheme 2 (Fig. 4Cc), the FWHM starts with low values, referring to a homogeneous $\varphi$ distribution in the region covered by the initial areas, selected from the lower border, and grows up to the last areas. This occurs due to the fact that larger areas comprise, progressively, more diversified $\varphi$ values. Scheme 3, in turn, provides a better defined plateau, satisfying the $10 \% R D$ in the initial areas. These differences found between schemes 2 and 3 are clearly portrayed in the CT image (Fig. $4 \mathrm{Ca}$ ) of this soil, in which regions with very different densities in the upper and lower borders are actually seen. 

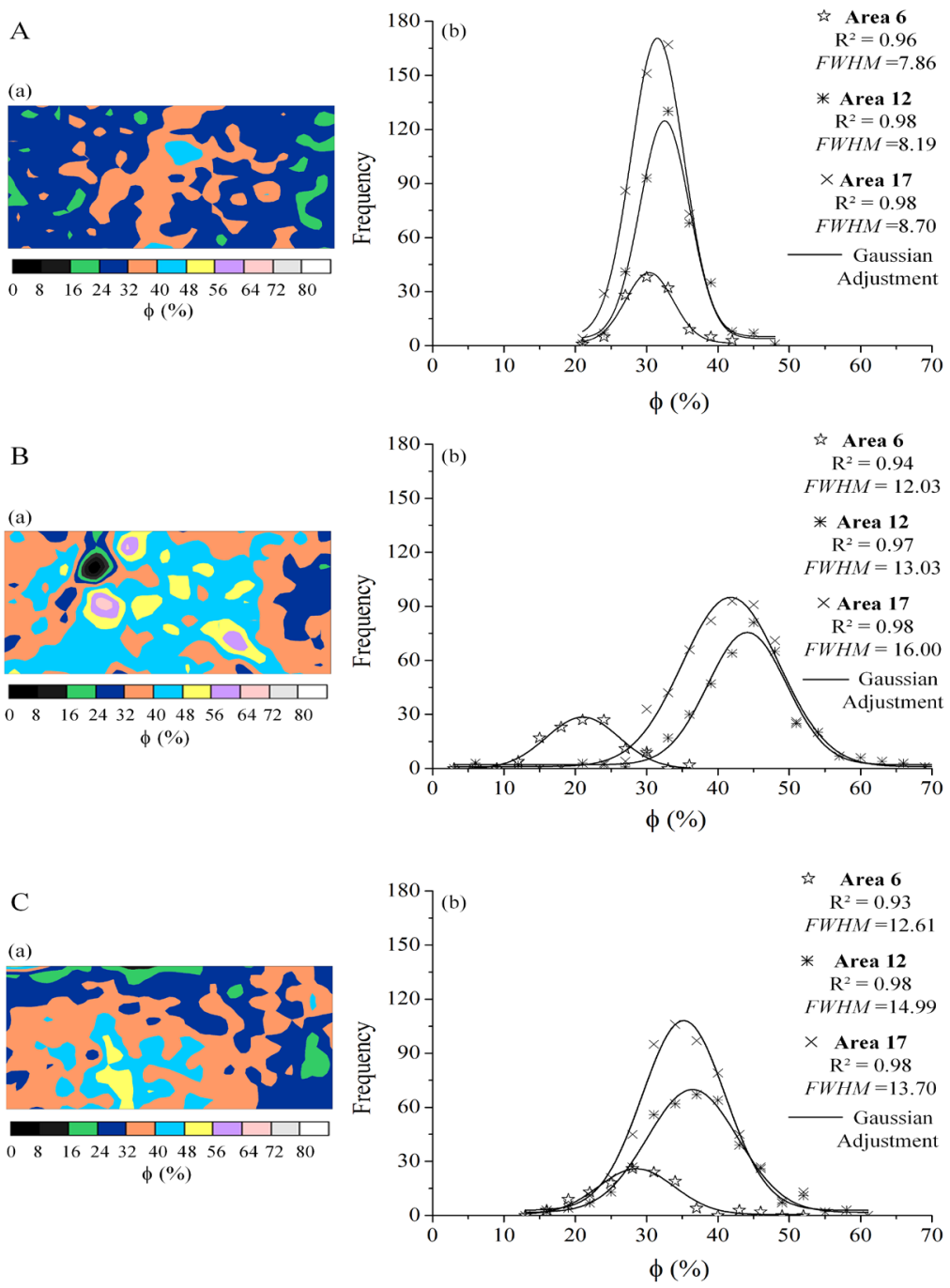
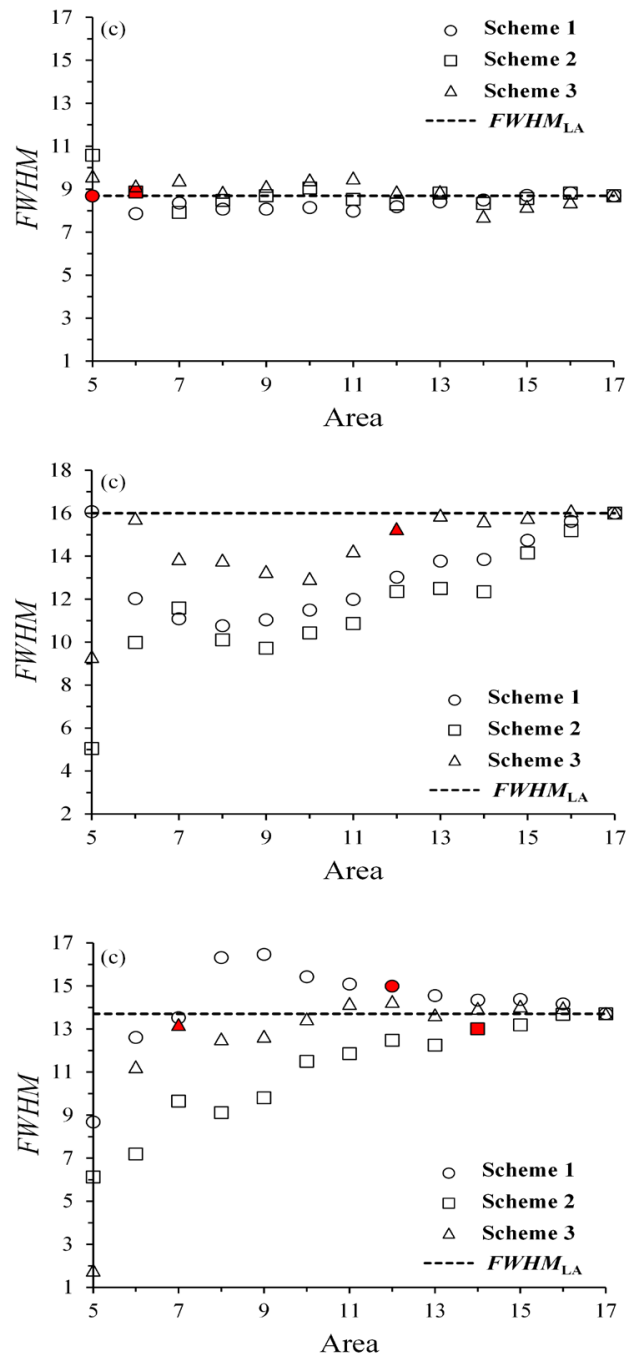

Fig. 4. Analyses carried out for one of the: A - Geric Ferralsol (GF), B - Rhodic Ferralsol (RF), and C - Eutric Nitosol (EN) soil samples: (a) CT image generated for the largest/last area (LA) selected inside the soil total porosity $(\varphi)$ matrix; (b) Graphs of frequency distribution for small (06), intermediate (12) and large (17) area sizes selected inside the matrix, according to scheme 1; (c) graphs of the full width at half maximum ( $F W H M$ ) as a function of the areas selected inside the matrix. The dotted line represents the $F W H M$ of LA, which is the same for all schemes; red filled icon represents the representative elementary area $(R E A)$ related to the $10 \%$ relative deviation.

It is important to consider that the $R E A$ represents the smallest area size suitable for the measurement of a specific parameter, and any area of a larger size could be considered equally valid. However, it is noticed that the REA is variable between the samples under analysis for a same $R D$ criterion, that is, there are few cases in which the specific $R D$ evaluated is reached in the same area for two samples, and this is even rarer for more than two. This fact can be explained taking into consideration the heterogeneity of the soil samples. Pires et al. (2005) analyzed different $T U$ values obtained within the same sample, divided into 15 adjacent areas. Those authors observed significant variation among $T U$, revealing variations in the soil structure, resulting from natural or artificial processes. Similar results were also observed by VandenBygaart and Protz (1999) and Baveye et al. (2002).
As an example, Table 3 shows the number of samples satisfying the $10 \% R D$ criterion according to the areas selected using schemes 1, 2 and 3 for the three soils. Following the representative elementary size concept, the $R E A$ admitted for evaluation of the $\varphi$ distribution corresponds to the smallest common area among the samples for which the considered $R D$ was reached. It means that, considering all samples, the last area for which the $R D$ criterion was satisfied is in fact the REA. The REA found for the GF, RF and EN soils was $514.3,514.3$ and $555.4 \mathrm{~mm}^{2}$ (scheme 1); 279.5, 393.3 and $457.4 \mathrm{~mm}^{2}$ (scheme 2) and 457.4, 457.4 and $457.4 \mathrm{~mm}^{2}$ (scheme 3).

It could be observed that, regardless of the area selection scheme, the $\varphi$ distribution REA for GF was lower than or even equal to that estimated in RF and EN (Table 3). This result indicates that GF soil has the most homogeneous 
T a b l e 3. Number of samples that reached the $10 \%$ relative deviation $(R D)$ criterion according to the areas selected inside the total soil porosity $(\varphi$ ) matrix using schemes 1, 2 and 3 for the Geric Ferralsol (GF), Rhodic Ferralsol (RF) and Eutric Nitosol (EN) soils. The blue highlighted fields represent the last area for which the considered $R D$ was reached, considering all samples

\begin{tabular}{|c|c|c|c|c|c|c|c|c|c|c|c|}
\hline \multirow[t]{2}{*}{ Area } & \multirow{2}{*}{$\begin{array}{c}\text { Size } \\
\left(\mathrm{mm}^{2}\right)\end{array}$} & \multicolumn{3}{|c|}{ Scheme 1} & \multirow{2}{*}{$\begin{array}{c}\text { Size } \\
\left(\mathrm{mm}^{2}\right)\end{array}$} & \multicolumn{3}{|c|}{ Scheme 2} & \multicolumn{3}{|c|}{ Scheme 3} \\
\hline & & GF & $\mathrm{RF}$ & $\mathrm{EN}$ & & GF & $\mathrm{RF}$ & EN & GF & $\mathrm{RF}$ & EN \\
\hline 5 & 98.0 & 2 & - & - & 54.5 & - & - & - & - & - & - \\
\hline 6 & 146.4 & 1 & - & - & 79.9 & 1 & - & - & - & - & 1 \\
\hline 7 & 204.5 & - & - & - & 110.1 & - & - & - & - & - & 1 \\
\hline 8 & 272.3 & 1 & 1 & - & 145.2 & - & - & - & - & - & - \\
\hline 9 & 349.7 & 1 & - & - & 185.1 & - & 1 & - & - & - & - \\
\hline 10 & 390.8 & - & - & - & 229.9 & - & - & 1 & 1 & - & - \\
\hline 11 & 432.0 & - & - & - & 279.5 & 3 & - & - & - & 2 & - \\
\hline 12 & 473.1 & - & 1 & 1 & 334.0 & - & - & - & 1 & 1 & - \\
\hline 13 & 514.3 & 1 & 2 & 3 & 393.3 & - & 2 & 2 & 2 & - & - \\
\hline 14 & 555.4 & - & - & 1 & 457.4 & - & - & 1 & 1 & 2 & 2 \\
\hline 15 & 596.5 & - & - & - & 526.4 & - & - & - & - & - & - \\
\hline 16 & 637.7 & - & - & - & 600.2 & - & - & - & - & - & - \\
\hline 17 & 678.8 & - & - & - & 678.8 & - & - & - & - & - & - \\
\hline \multirow[t]{2}{*}{ Total \% } & 6 & 4 & 5 & & 4 & 3 & 4 & 5 & 5 & 4 & \\
\hline & 100 & 67 & 83 & & 67 & 50 & 67 & 83 & 83 & 67 & \\
\hline
\end{tabular}

structure when compared to the other soils under study (Fig. 5), which is related mainly to the fact that this is a sandy soil (Jury and Horton, 2004). According to Bouma (1983), the $R E A$ should increase when the texture is finer and the soil is more structured. In addition, the $R E A$ tends to be larger in strongly aggregated, rocky and cracked soils, that is, more heterogeneous soils (Hillel, 1998).

Besides the texture, another explanation for the differences observed among soils is related to their management. The GF soil was collected from an area which has not been submitted to tillage and which was covered by grass. The presence of fine roots and a small number of biopores were observed in its samples. For the RF soil, the heterogeneity found is due to the fact that the samples were collected in a forest area with a large number of roots, leaves and soil fauna. The presence of a large number of biopores inside the RF samples was observed. Finally, the EN samples were collected in the inter-row of a coffee plantation. The area was submitted to traffic for weed control and harvesting. Some compaction was observed in the samples due to the management of the experimental area.

It was also seen that different schemes provided different REAs for the same soil. The biggest REAs were determined for scheme 1 (Table 3 ). This reveals that the growth of areas starting at the center of the $\varphi$ matrices (Fig. 3f) is more effective to detect the $\varphi$ distribution heterogeneities over the soil sample. This means that the use of small areas, such as that of scheme $2-\mathrm{GF}$ $\left(279.5 \mathrm{~mm}^{2}\right)$, randomly selected inside a CT image might lead to an inccurate evaluation of the $\varphi$ distribution. Such result indicates that according to the method of $R E A$ definition, the evaluation of a specific soil physical property based on the size found as representative, can under or overestimate the values of this property.

When only a small number of samples satisfy certain $R D$ (Table 3 ), this means that the size of the selected areas inside the samples is not enough for the variations to occur within the limit established. In these cases, it would be interesting that the REA study was carried out for a larger number of samples, or even with larger samples.

It is important to emphasize that the concept of representative elementary sizes must be used cautiously, since the distinct parameters might exhibit different space and time patterns, requiring samples with different length, area or volume to obtain their representative measure. In statistical terms, the variation of the property under analysis decreases when the sample size increases (Starr et al., 1995). However, taking 'structured' domains, that is, domains that vary systematically in a certain direction, increasing the sample size might not produce a representative result (Baveye et al., 2002; Hillel, 1998). 
a

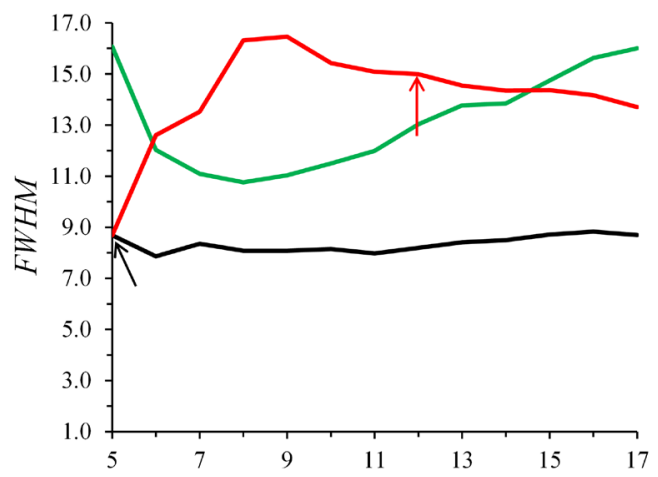

$\mathrm{b}$

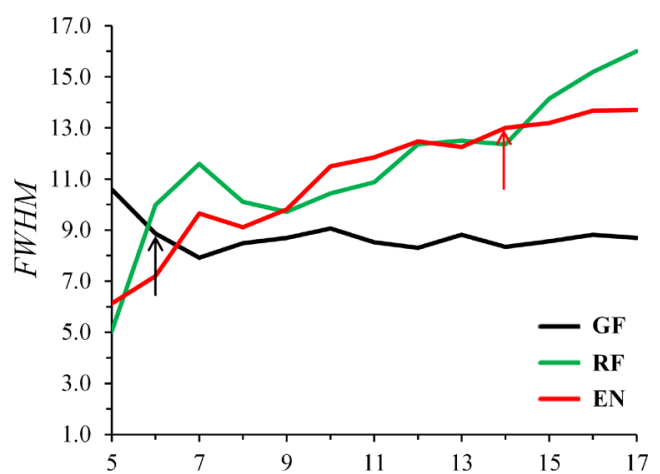

$\mathrm{c}$

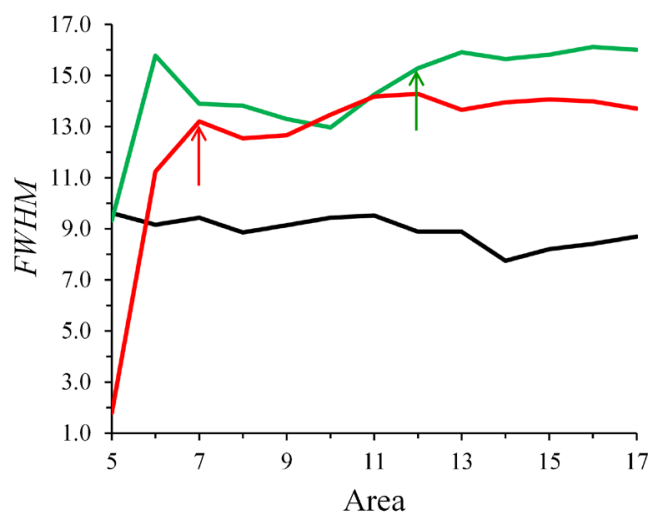

Fig. 5. Graphs of the full width at half maximum (FWHM) as a function of the areas selected inside the total soil porosity $(\varphi)$ matrix for comparison between the FWHM behaviours according to the soil texture and each studied scheme: a - scheme $1, \mathrm{~b}-$ scheme 2, c- scheme 3. Geric Ferralsol (GF) is a sandy soil while Rhodic Ferralsol (RF) and Eutric Nitosol (EN) are clayey soils. The arrows delimit the area for which the $10 \%$ relative deviation $(R D)$ was reached for the same samples investigated in Fig. 4.

\section{CONCLUSIONS}

1. Smaller representative elementary area sizes were obtained for a non-cultivated sandy clay loam soil in comparison to two different clayey soils, one of them under mixed forest and the other submitted to cultivation, meaning that soil texture has a major effect on representative elementary area.

2. When comparing only the studied clayey soils, the representative elementary area determined for the soil under forest was smaller than that for the cultivated soil, considering two evaluated schemes of area selection, which indicates that not only soil texture has an effect on representative elementary sizes, required for a soil parameter measurement, but also soil management can present influence.

3. Different schemes of area selection provided different representative elementary areas for the same soil, suggesting that the heterogeneity over the soil sample depth should be considered to define the representative elementary area.

4. The biggest representative elementary areas were determined for scheme 1 . This reveals that the growth of areas starting at the center of the soil porosity matrices is more effective to detect the soil porosity distribution heterogeneities over the soil sample.

Conflict of interest: The Authors do not declare conflict of interest.

\section{REFERENCES}

Baveye P. and Boast C.W., 1999. Physical scales and spatial predictability of transport processes in the environment. In: Assessment of Non-Point Source Pollution in the Vadose Zone (Eds D.L. Corwin, K. Loague, T.R. Ellsworth). American Geophysical Union, Washington, USA, 261-280.

Baveye P., Rogasik H., Wendroth O., Onasch I., and Crawford J.W., 2002. Effect of sampling volume on the measurement of soil physical properties: simulation with x-ray tomography data. Meas. Sci. Technol., 13, 775-784.

Bear J., 1972. Dynamics of Fluids in Porous Media. Dover Publications, New York, USA.

Bear J. and Cheng A.H-D., 2010. Modeling Groundwater Flow and Contaminant Transport. In: Theory and Applications of Transport in Porous Media, Springer, Heidelberg, Germany.

Borges J.A.R. and Pires L.F., 2012. Representative elementary area (REA) in soil bulk density measurements through gamma ray computed tomography. Soil Till. Res., 123, 43-49.

Borges J.A.R., Pires L.F., and Pereira A.B., 2012. Computed tomography to estimate the representative elementary area for soil porosity measurements. Sci. World J., 10, https:// www.hindawi.com/journals/tswj/2012/526380/cta/

Bouma J., 1983. Use of soil survey data to select measurement techniques for hydraulic conductivity. Agric. Water Manag., 6, 177-190.

Crestana S., Cruvinel P.E., Vaz C.M.P., Cesareo R., Mascarenhas S., and Reichardt K., 1992. Calibration and use of a computerized tomography in soil science (in Portuguese). R. Bras. Ci. Solo, 16, 161-167.

Cruvinel P.E., Cesareo R., Crestana S., and Mascarenhas S., 1990. $X$ - and $\gamma$-rays computerized minitomograph scanner for soil science. IEEE Trans. Instrum. Meas., 39, 745-750.

FAO/ISRIC/ISSS, 1998. World reference base for soil resource (No. 84). Food and Agriculture Organization of the United States, FAO, ISRIC and ISSS, Rome.

Ferraz E.S.B. and Mansell R.S., 1979. Determining water content and bulk density of soil by gamma ray attenuation methods. IFAS, Florida, USA. 
Ferreira T.R., Borges J.A.R., and Pires L.F., 2015. Representative elementary area for soil bulk density measurements of samples collected in volumetric rings by CT image analyses. Soil Till. Res., 152, 74-84.

Hillel D., 1998. Environmental Soil Physics: Fundamentals, Applications, and Environmental Considerations. Academic Press, London, ENG.

Jury W.A. and Horton R.C.N., 2004. Soil Physics. John Wiley, Hoboken, NJ, USA.

Kak A.C. and Slaney M., 1999. Principles of Computerized Tomographic Imaging. IEEE Press, New York, USA.

Knoll G.F., 2010. Radiation Detection and Measurement. Wiley, New York, USA.

Lal R. and Shukla M.K., 2004. Principles of Soil Physics. Marcel Dekker, New York, USA.

Microvis, 2000. Program Manual for Reconstruction and Visualization of tomographic imag (in Portuguese). EMBRAPA Agricultural Instrumentation, São Carlos, SP, BR.

Pedrotti A., Pauletto E.A., Crestana S., Holanda F.S.R., Cruvinel P.E., and Vaz C.M.P., 2005. Evaluation of bulk density of Albaqualf soil under different tillage systems using the volumetric ring and computerized tomography methods. Soil Till. Res., 80, 115-123.

Pires L.F., Bacchi O.O.S., Reichardt K., and Timm L.C., 2005. Application of gamma-ray computed tomography to analysis of soil structure before density evaluations. Appl. Radiat. Isot., 63, 505-511.
Pires L.F., Borges J.A.R., Bacchi O.O.S., and Reichardt K., 2010. Twenty-five years of computed tomography in soil physics: A literature review of the Brazilian contribution. Soil Till. Res., 110, 197-210.

Pires L.F., Cássaro F.A.M., Bacchi O.O.S., and Reichardt K., 2011a. Gamma-ray computed tomography in soil science: some applications. In: Computed Tomography - Special Applications (Ed. L. Saba). InTech., 293-318.

Pires L.F., Arthur R.C.J., Bacchi O.O.S., and Reichardt K., 2011b. Representative gamma-ray computed tomography calibration for applications in soil physics. Braz. J. Phys., 41, 21-28.

Starr J.L., Parkin T.B., and Meisinger J.J.,1995. Influence of sample size on chemical and physical soil measurements. Soil Sci. Soc. Am. J., 59, 713-718.

Timm L.C., Pires L.F., Reichardt K., Roveratti R., Oliveira J.C.M. and Bacchi O.O.S., 2005. Soil bulk density evaluation by conventional and nuclear methods. Aust. J. Soil Res., 43, 97-103.

Turner J.E., Downing D.J., and Bogard J.S., 2012. Statistical Methods in Radiation Physics. Wiley-VCH, Weinheim, Germany.

VandenBygaart A.J. and Protz R., 1999. The representative elementary area (REA) in studies of quantitative soil micromorphology. Geoderma, 89, 333-346.

Vaz C.M.P., Crestana S., Mascarenhas S., Cruvinel P.E., Reichardt K., and Stolf R., 1989. Using a computed tomography miniscanner for studying tillage induced soil compaction. Soil Technol., 2, 313-321. 